\title{
Melting of incommensurate-ferroelectric phase with magnetic field in multiferroic $\mathrm{TbMnO}_{3}$
}

\author{
D. N. Argyriou, ${ }^{1}$ N. Aliouane, ${ }^{1}$ J. Strempfer, ${ }^{2}$ I. Zegkinoglou, ${ }^{2}$ B. Bohnenbuck, ${ }^{2}$ K. Habicht, ${ }^{1}$ and M. v. Zimmermann ${ }^{3}$ \\ ${ }^{1}$ Hahn-Meitner-Institut, Glienicker Strasse 100, Berlin D-14109, Germany \\ ${ }^{2}$ Max-Planck-Institut für Festkörperforschung, Heisenbergstraße 1, 70569 Stuttgart, Germany \\ ${ }^{3}$ Hamburger Synchrotronstrahlungslabor HASYLAB at Deutsches Elektronen-Synchrotron DESY, Notkestrasse 85, \\ 22603 Hamburg, Germany
}

(Received 17 August 2006; published 4 January 2007)

\begin{abstract}
We have investigated the reentrant ferroelectric behavior of $\mathrm{TbMnO}_{3}$ under a $c$-axis-aligned magnetic field $(H \| c)$ using neutron and x-ray single-crystal diffraction. We find that below $T_{N}=41 \mathrm{~K}$ and at high field the incommensurate ordering of $\mathrm{Mn}$ spins melts and coincides with the disappearance of ferroelectricity. The paraelectric phase which emerges is characterized by a simple commensurate antiferomagnetic ordering. We suggest that the reentrance of ferroelectricity at low temperatures and high magnetic field coincides with the development of incommensurate $\mathrm{Mn}$ - and $\mathrm{Tb}$-spin ordering.
\end{abstract}

DOI: 10.1103/PhysRevB.75.020101

PACS number(s): 77.22.Ej, 77.80.Bh, 75.25.+z

\section{INTRODUCTION}

Although in conventional ferroelectrics the requirements of magnetism and ferroelectricity are chemically incompatible, ${ }^{1}$ recent investigations of spin-frustrated maganite perovskites $R \mathrm{MnO}_{3}$ have shown that this chemical incompatibility can be overcomed by frustration. ${ }^{2,3}$ For $\mathrm{LaMnO}_{3}$ the antiferro-type ordering of singly occupied $\mathrm{Mn}^{3+}$ $3 d_{3 z^{2}-r^{2}}$ orbitals produces a ferromagnetic (FM) interaction that results in A-type magnetic structure-an antiferromagnetic stacking of ferromagnetic sheets. As the size of the trivalent $R$ cation decreases, the $A$-type phase becomes frustrated, highlighted by the steady decrease of $T_{N}$ from $150 \mathrm{~K}$ for $R=\mathrm{La}$ to $\sim 40 \mathrm{~K}$ for $R=\mathrm{Eu} .^{3}$ This behavior is ascribed to a weakening of nearest-neighbor FM interactions. ${ }^{3,4}$ For $R=\mathrm{Gd}, \mathrm{Tb}$, and $\mathrm{Dy},{ }^{5,6}$ the frustration leads to a complex magnetic structure which couples strongly to a ferroelectric polarization. Although the size of the polarization is relatively small $\left(\sim 0.05 \mu \mathrm{C} / \mathrm{cm}^{2}\right)$, its direction is field dependent.

For $R=\mathrm{Tb}$ in particular, at $T_{N} \sim 41 \mathrm{~K}$ the $A$-type phase is replaced by a longitudinal spin density wave (SDW) propagating along the $b$ direction with wave vector $\kappa_{m}^{\mathrm{Mn}}=\left(0, k \pm \delta_{m}^{\mathrm{Mn}}, 0\right), \quad \delta_{m}^{\mathrm{Mn}} \sim 0.29 .{ }^{6}$ With further cooling $\delta_{m}^{\mathrm{Mn}}$ decreases somewhat until a second transition into a spiral phase at $T_{\mathrm{C}}=28 \mathrm{~K}$. This magnetic ordering corresponds to an elliptic cycloid with the spins rotating around the $a$ axis. $^{7}$ Coincident with the transition to a spiral phase a spontaneous electric polarization parallel to the $c$ axis $(P \| a)$ is found. ${ }^{2}$ Accompanying the magnetic ordering there is a lattice modulation with $\delta_{l}=2 \delta_{m}$ (second harmonic).

In addition to the ordering of $\mathrm{Mn}$ spins, $\mathrm{Tb}$ spins also contribute to the magnetism. At $15 \mathrm{~K}$, Tb spins are induced to order along the $a$ axis with the same periodicity as $\mathrm{Mn}$ spins. ${ }^{7}$ However, below $T_{N}^{\mathrm{Tb}}=7 \mathrm{~K}$, the appearance of new incommensurate (IC) reflections is found to coincide with a peak in the specific heat. ${ }^{2}$ From the size of this peak and the magnitude of the magnetic neutron intensities their origin has been ascribed to a sinusoidal $\mathrm{Tb}$-spin ordering with wave vector $\kappa_{m}^{\mathrm{Tb}}=\left(0, k \pm \delta^{\mathrm{Tb}}, 0\right), \delta^{\mathrm{Tb}}=0.42 .{ }^{9}$

Considerations based on Landau theory have demonstrated a coupling of a uniform polarization $\mathbf{P}$ to an inhomo- geneous magnetization $\mathbf{M},{ }^{7,10}$ so that $\mathbf{P}=\gamma \chi M_{b} M_{c}[\mathbf{a} \times \kappa]$, where $\gamma$ and $\chi$ are coupling constants and the susceptibility, $M_{b}$ and $M_{c}$ are magnetic moments along the $b$ and $c$ axes, and $\mathbf{a}$ is the axis of rotation of $\mathrm{Mn}$ spiral. ${ }^{10}$ This relation would predict that $\mathbf{P}$ lies along the $c$ axis as indeed is observed experimentally.

When a magnetic field is applied to $\mathrm{TbMnO}_{3}$ along the $a$ or $b$ axis the polarization flops from $P \| c$ to $P \| a^{2}$ driven by a change in the rotation axis of the spiral from the $a$ direction $(P \| c)$ to the $c$ direction $(P \| a)$. However, a strikingly different behavior is observed when a magnetic field is applied along the $c$ axis $(H \| c) .{ }^{6}$ Here a reentrant behavior is found: as illustrated in Fig. 1, cooling the sample below $T_{\mathrm{C}}$ in $H \|_{c} \gtrsim 8 \mathrm{~T}$ results in the melting of the ferroelectric phase at about $20 \mathrm{~K}(8 \mathrm{~T})$ but at lower temperature the ferroelectric-

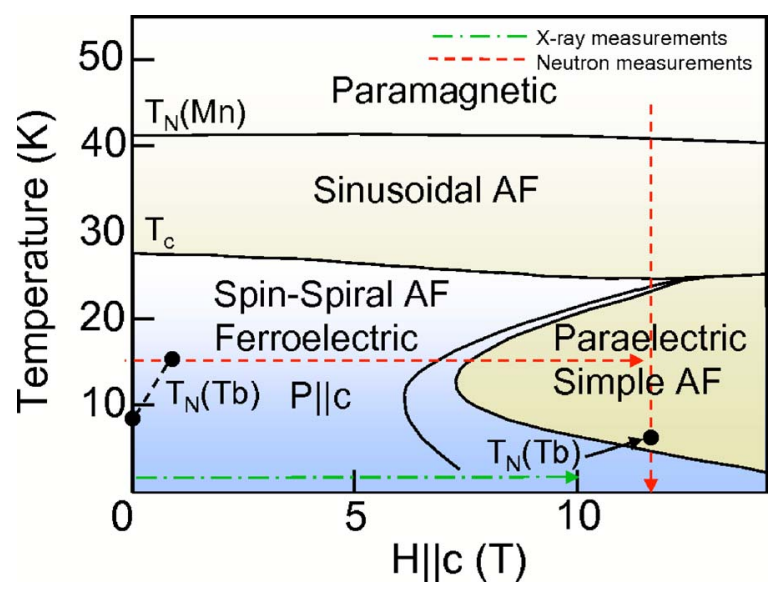

FIG. 1. (Color online) Magnetoelectric phase diagram of $\mathrm{TbMnO}_{3}$ for $H \| c$, reproduced from Ref. 6. The $H-T$ phase diagram shows the high-temperature paramagnetic, lower-temperature sinusoidal antiferromagnetic (AF) regions. The onset of ferroelectric polarization at $T_{\mathrm{C}}$ as a function of $H$ and $T$ is also shown. The two lines at the phase boundary to the high-field paraelectric phase represent hysteresis. The observation of $T_{N}^{\mathrm{Tb}}$ from neutron diffraction is also indicated in the figure as solid circles $(\bullet)$. The dashed and dot-dashed lines indicate the direction of the neutron and x-ray diffraction measurements, respectively. 

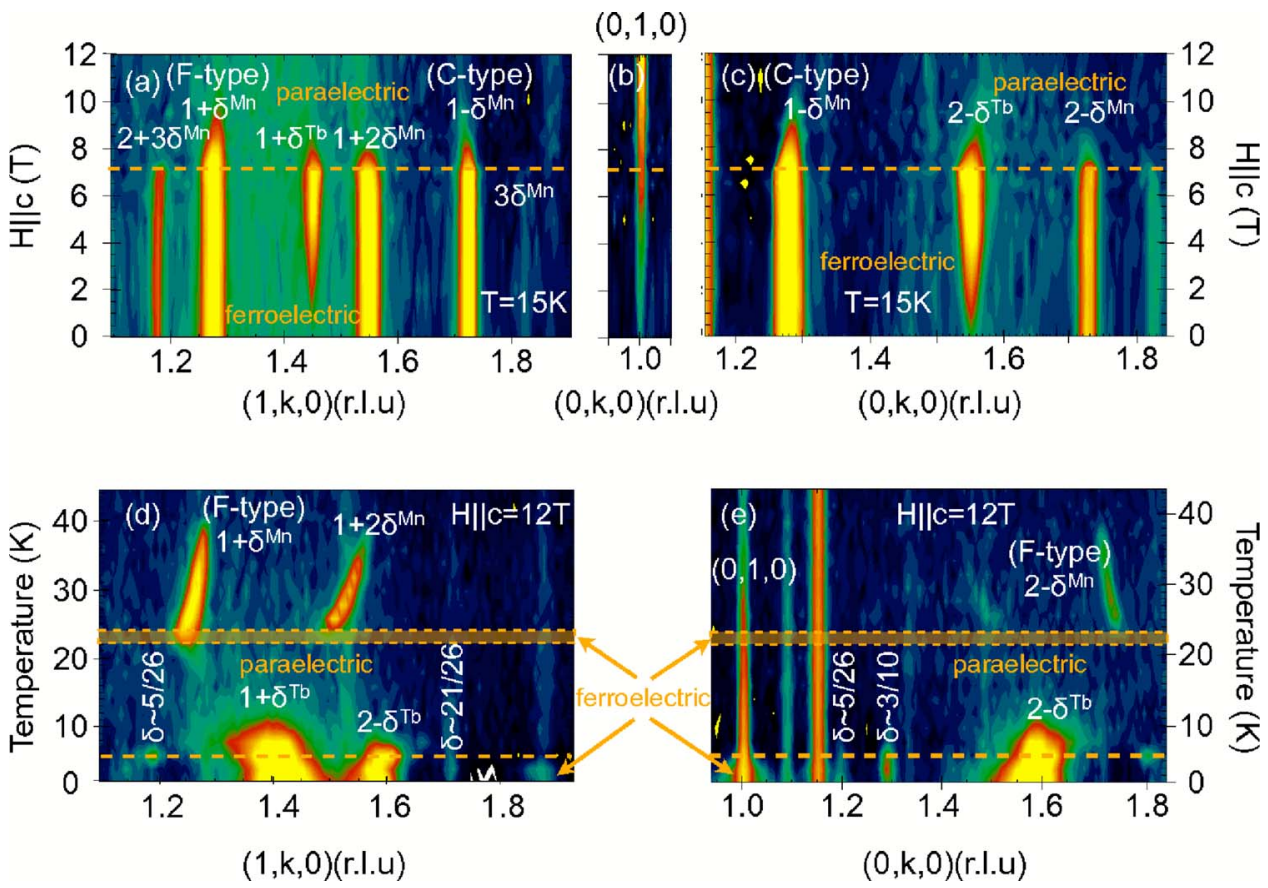

FIG. 2. (Color online) A two-dimensional plot representing neutron diffraction scans measured on the FLEX spectrometer along $(0, k, 0)$ and $(1, k, 0)(\mathrm{a})-(\mathrm{c})$ as a function of magnetic field $H \| c$ at $15 \mathrm{~K}$ and (d), (e) as a function of temperature in a magnetic field $H \| c=12 \mathrm{~T}$. Indicative magnetic reflections for $\mathrm{Mn}$ and $\mathrm{Tb}$ spin order are labeled. For (b) the scan range is $(0,1 \pm 0.07,0)$. Transitions to paraelectric and ferroelectric states from Fig. 1 are also represented here.

ity emerges again. Such behavior is not predicted by the recent phenomenological modeling. ${ }^{10}$

To shed light on the reentrant behavior for $H \| c$ in $\mathrm{TbMnO}_{3}$ we have used in-field neutron and synchrotron $\mathrm{X}$-ray single-crystal diffraction to measure the field response of the magnetic and crystal structure up to $14 \mathrm{~T}$. Neutron diffraction experiments were made on a large single crystal of $\mathrm{TbMnO}_{3},{ }^{11}$ at the BENSC facility of the Hahn-Meitner Institut, Berlin. For measurements with $H \|_{c}$ we used the FLEX cold triple-axis spectrometer with collimation of $40^{\prime}$ $40^{\prime}-40^{\prime}, k_{i}=1.5 \AA^{-1}$, and a cooled Be filter was positioned in the incident beam, while a magnetic field was applied using the vertical 14.5-T superconducting magnet VM1. Measurements were made with the analyzer set to $E=0 \mathrm{meV}$. The sample was mounted with the $c$ axis vertical allowing the measurements of reflections in the $a b$ plane. Here only the $F$ and $C$-type reflections are accessible with the following selection rules: $F(h+k=$ even and $l=$ even $)$ and $C(h+k=$ odd and $l=$ even). The $A$ - and $G$-type reflections lie on the next layer of reciprocal space with $l=1$ (selection rules: $A$ ( $h+k=$ even with $l=$ odd $)$ and $G(h+k=$ odd with $l=$ odd $)$ and could not be observed in the neutron measurements. The synchrotron $\mathrm{X}$-ray measurements were carried out at beamline BW5 at HASYLAB at a photon energy of $100 \mathrm{keV}$, using a 10-T superconducting magnet with horizontal field. The sample was cut from the same boule as the crystal used for the neutron experiment with a thickness close to the absorption length of $\mathrm{TbMnO}_{3}$ at $100 \mathrm{keV}(0.6 \mathrm{~mm})$. The experiment was conducted in Laue geometry with the magnetic field applied along the $c$ axis and the $b c$ plane lying in the horizontal scattering plane.

\section{RESULTS: NEUTRON DIFFRACTION}

In Fig. 2 we show diagrams of the neutron diffraction data of cuts through reciprocal space as a function of either magnetic field or temperature. The sample was first cooled in zero field to $15 \mathrm{~K}$, and the field $(H \| c)$ was ramped from 0 up to $12 \mathrm{~T}$ [Figs. 2(a) and 2(b)]. In zero field, we find the first harmonic reflections ( $F$ and $C$ type) due to the IC ordering of $\mathrm{Mn}$ spins as well as the second harmonic reflection $\left(1,1+2 \delta^{\mathrm{Mn}}, 0\right)$. At $T=15 \mathrm{~K}$, the application of a magnetic field does not induce significant changes in the magnitude of the wave vector or in the intensity of these reflections up to $7 \mathrm{~T}$ as shown in Figs. 3(a) and 3(b). Similarly, the electrical spontaneous polarization is weakly affected by $H \leqslant 7 \mathrm{~T}^{6}$ At $H \sim 1.5 \mathrm{~T}$, Tb spins begin to order antiferromagnetically as indicated by the observation of $\left(1,1+\delta^{\mathrm{Tb}}, 0\right)$ and $\left(0,2-\delta^{\mathrm{Tb}}, 0\right)$ reflections [Figs. 2(a), 2(c), and 3(a)] with $\delta^{\mathrm{Tb}} \sim 0.45$. In addition, with increasing field at this temperature a simple antiferromagnetic (AF) ordering is induced as indicated by the observation of the $(0,1,0)$ reflection and an increase of its intensity with increasing field [Figs. 2(b) and 3(a)]. Above the critical field of $H_{c} \sim 7 \mathrm{~T}$, the intensity of all IC magnetic reflections due to $\mathrm{Mn}$ - and $\mathrm{Tb}$-spin ordering is strongly suppressed and disappear with increasing field [see Fig. 3(a)]. Simultaneously the polarization of $\mathrm{TbMnO}_{3}$ disappears, indicating a transition from a ferroelectric to a paraelectric phase ${ }^{6}$ (see Fig. 1).

As we increase the field above $H_{C}$ the value of $\delta^{\mathrm{Mn}}$ increases from 0.272 up to 0.288 [Fig. 3(b)], while the FWHM of the $C$ - and $F$-type reflections also increases [see Fig. 3(c)], indicating that the IC magnetic structure is destabilized. In 


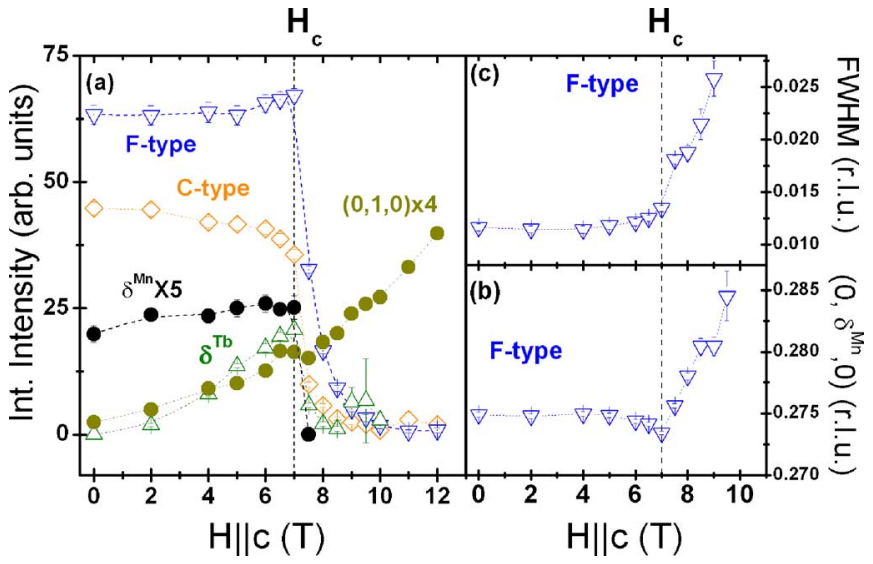

FIG. 3. (Color online) Results of neutron diffraction measurements at $T=15 \mathrm{~K}$ as a function of $H \| c$. (a) Field dependence of the integrated intensity of characteristic magnetic and structural reflections arising from $\mathrm{Mn}$ - and $\mathrm{Tb}$-spin ordering as well as the $(0,1,0)$ reflection. The $F$ - and $C$-type $3 \delta^{\mathrm{Mn}}, 2 \delta^{\mathrm{Mn}}$, and $\delta^{\mathrm{Tb}}$ labels in the figure correspond, respectively, to $\left(1,1+\delta^{\mathrm{Mn}}, 0\right),\left(1,2-\delta^{\mathrm{Mn}}, 0\right)$, $\left(1,2-3 \delta^{\mathrm{Mn}}, 0\right),\left(1,2 \delta^{\mathrm{Mn}}, 0\right)$, and $\left(1, \delta^{\mathrm{Tb}}, 0\right)$. (b), (c) Field dependence of the wave vector $\delta^{\mathrm{Mn}}$ and full width at half maximum (FWHM) of the $\left(1,1+\delta^{\mathrm{Mn}}, 0\right)$ reflection at $T=15 \mathrm{~K}$.

sharp contrast, the intensity of the AF (010) reflection steadily increases across this transition while it displays a small anomaly at $H_{c}$. Our measurements demonstrate that this high-field paralelectric phase is characterized by a simple AF ordering and the absence of any IC antiferromagnetic ordering arising from either $\mathrm{Mn}$ or Tb spins.

In Figs. 2(d) and 2(e) we show field-cooled neutron diffraction measurements with $H \| c=12 \mathrm{~T}$ for the same cuts through reciprocal space. Here Mn spins first order incommensurately as indicated by the observation of the $F$-type $\delta^{\mathrm{Mn}} \sim 0.278$ and $2 \delta^{\mathrm{Mn}}$ reflections. At this field value the $C$-type reflection was not observed. The $\mathrm{Mn}$ wave vector smoothly decreases with temperature to the commensurate value of $\frac{1}{4}$ at $23 \mathrm{~K}$ [see Fig. 4(a)]. Indeed ferroelectricity is reported to occur in this region over a brief temperature window between 25 and 22 K Ref. 6 (Fig. 1). While the intensity of the $F$-type reflection and its second harmonic increases on cooling to $\sim 26 \mathrm{~K}$, below this temperature the intensity of these reflections is suppressed and they completely disappear below $T \sim 22 \mathrm{~K}$ [see Figs. 2(d), 2(e), and 4(b)]. The disappearance of the IC Mn reflections coincides with the melting of the ferroelectric phase, while over the same region in temperature the intensity of $(0,1,0)$ reflection steadily increases on cooling [Figs. 2(d) and 4(a)]. Between 20 and $12 \mathrm{~K}$, in the paraelectric state, we find no evidence of incommensurate reflections, which confirms again that the paraelectric state is characterized by a simple $\mathrm{AF}$ phase [see Figs. 2(a)-2(c)].

Below $T_{N}^{\mathrm{Tb}}=8 \mathrm{~K}$ the incommensurate ordering of $\mathrm{Tb}$ spins is observed by reflections with $\delta^{\mathrm{Tb}} \sim 0.42$ at $2 \mathrm{~K}$, a value similar to that found in zero field. The Tb-spin ordering appears to influence the coherence length of the simple AF phase as indicated by the increase of the width of the $(0,1,0)$ reflection [Figs. 2(c) and 4(c)]. For $T<7 \mathrm{~K}$, additional reflections with $\delta \sim 5 / 26$ and $\delta \sim 3 / 10$ are observed indicative

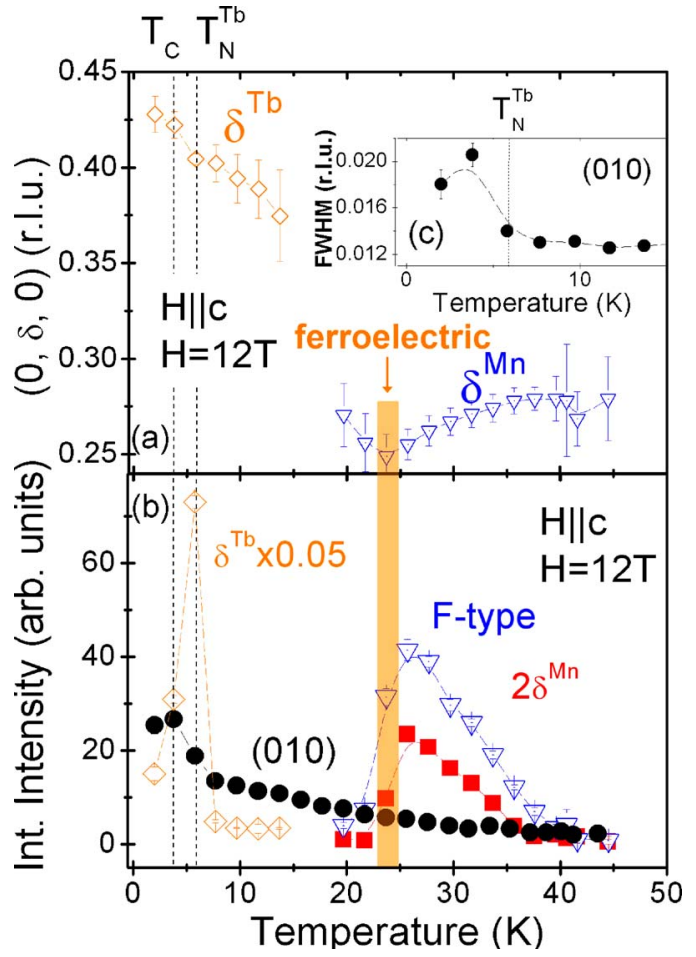

FIG. 4. (Color online) Results of field-cooled neutron diffraction measurements with $H \|_{c}=12 \mathrm{~T}$. (a) Temperature dependence of $\delta^{\mathrm{Mn}}$ and $\delta^{\mathrm{Tb}}$. (b) Temperature dependence of the integrated intensity of characteristic magnetic and structural reflections arising from Mnand Tb-spin ordering as well as the $(0,1,0)$ reflection. The labels here are as in Fig. 3(a). (c) Temperature dependence of the FWHM of the $(0,1,0)$ reflection. The brief region in which ferroelectricity appears at high temperature is highlighted, while $T_{\mathrm{C}}=5 \mathrm{~K}$ indicates the reentrance of ferroelectrocity at lower temperatures.

of a complex spin arrangement and magnetoelastic coupling [Figs. 2(d) and 2(e)].

The ordering of $\mathrm{Tb}$ spins in the field-cooled measurement shows a complex behavior which is illustrated in the scans through the $(1,1.42,0)$ reflection [Fig. 5(a)]. On cooling through $T_{N}^{\mathrm{Tb}}$, this reflection shows initially a weak broad signal at $8.1 \mathrm{~K}$ and splits into what appears to be two separate reflections at $7.8 \mathrm{~K}$. Although there is a sharp rise in the intensity of this reflection with further cooling, the line shape develops a complex character, possibly arising from two distinct peaks. This unusual behavior in the ordering of $\mathrm{Tb}$ ions correlates with the reentrance of ferroelectricity at $T_{\mathrm{C}}=5 \mathrm{~K}$ (Ref. 6) and the broadening of the $(0,1,0)$ reflection at the same temperature [see Figs. 2(e) and 4(d)].

\section{X-RAY DIFFRACTION}

To clarify this behavior we performed higher-resolution in-field synchrotron diffraction experiments at $2 \mathrm{~K}$, while field was applied after zero-field cooling. At this temperature the ferroelectric phase of $\mathrm{TbMnO}_{3}$ remains stable up to a field of $10 \mathrm{~T},{ }^{6}$ the maximum value reached in this measurement. In this geometry we could track the first and second harmonic Mn $A$-type reflections $\left(0, \delta^{\mathrm{Mn}}, 5\right)$ and $\left(0,2 \delta_{m}, 5\right)$. 

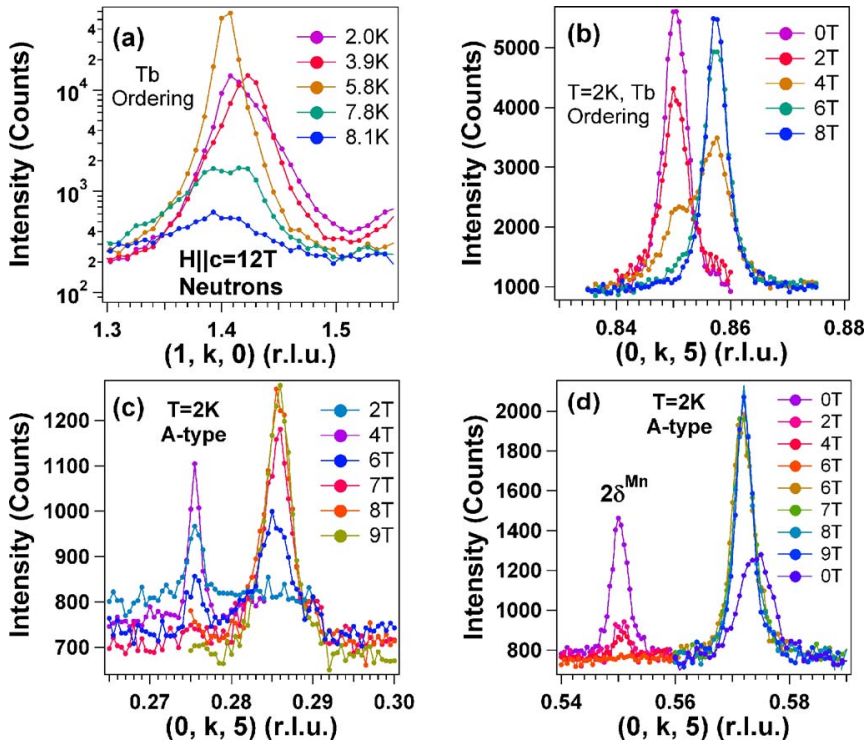

FIG. 5. (Color online) (a) Neutron diffraction scans measured on the FLEX spectrometer along the $(1, k, 0)$ direction at constant field $(H \| c=12 \mathrm{~T})$. (b)-(d) X-ray diffraction scans at constant temperature $(T=2 \mathrm{~K})$ along the $(0, k, 5)$ direction for different fields $(H \| c)$.

For field values above $2 \mathrm{~T}$ the first harmonic is visible while increasing field suppresses the intensity of both reflections as shown in Figs. 5(c) and 5(d). At $6 \mathrm{~T}$ there are two reflections present at $\delta^{\mathrm{Mn}}=0.275$ and 0.285 (and similarly for $2 \delta^{\mathrm{Mn}}$ ), while for higher field only the higher $\delta^{\mathrm{Mn}}$ reflection is visible which gains in intensity as $H$ increases. Such a discontinuous jump in the wave vector is also found for the $\left(0,2 \delta^{\mathrm{Tb}}, 5\right)$ reflection where $\delta^{\mathrm{Tb}}$ jumps from 0.85 to 0.86 at $4 \mathrm{~T}$. Multiple scattering as the origin of these IC reflections can be excluded since this would only appear at integer positions in reciprocal space. These $\mathrm{x}$-ray measurements suggest that the application of a field results in a sharp magnetoelastic transition for both $\mathrm{Mn}$ and $\mathrm{Tb}$ sublattices at different critical fields and correlates well with physical property measurements. For example, $M$ vs $H$ data and magnetocapacitance measurements for $H \| c$ at $4 \mathrm{~K}$ indicate the opening of a hysteresis between $\sim 4 \mathrm{~T}$ and $13 \mathrm{~T}$ (Ref. 3). Our measurements of the discontinuous jumps in $\delta^{\mathrm{Mn}}$ and $\delta^{\mathrm{Tb}}$ correlate well with these observations.

\section{DISCUSSION}

The behavior of the magnetic structure of $\mathrm{TbMnO}_{3}$ with applied field can be understood in terms of the degrees of freedom of the Mn-spiral phase. These involve precessions of the spiral-rotation axis around the $b$ or $c$ axis, which produce components of the spiral perpendicular to the $b c$ plane, while a third mode describes changes of phase along the $b$ axis (phason mode). ${ }^{12,13}$ For $H \| a$ or $H \| b$ the field couples to the precessions of the spiral-rotation axis ${ }^{10}$ to the flop of the spiral axis and thus $\mathbf{P} .{ }^{12}$ However, there is no appropriate degree of freedom that would result in a flop of the spiral rotation axis for $H \| c$, consistent with the calculated $H-T$ phase diagram for $\mathrm{TbMnO}_{3} \cdot{ }^{10}$ Here only a suppression of $T_{\mathrm{C}}$ with increasing field is predicted. ${ }^{10}$ In agreement with this picture our measurements show that the Mn spiral is unstable for $H \| c$ and the field leads to its melting in preference for a simple antiferromagnetic phase.

What is surprising in this context is that the behavior of the polarization is reentrant. Our x-ray measurements at $2 \mathrm{~K}$ indicate that the Mn spiral is stable up to field values of $10 \mathrm{~T}$ as indicated by the observation of the $A$-type reflection, consistent with the phase diagram of Fig. 1. At sufficiently high fields and low temperature the reemergence of the spiral ordering appears to coincide with that of $\mathrm{Tb}$ spins, which have a much larger moment. This suggests the possibility that $\mathrm{Tb}$ ordering may indeed stabilize the Mn spiral in this regime. To confirm this behavior it would be helpful to expand the phenomenological treatment of $R$ spins to include coupling terms between $\mathrm{Mn}$ and $R$ spins.

\section{CONCLUSIONS}

The measurements we present here show that application of a field along the $c$ axis melts the IC magnetic ordering of Mn spins which coincides with the suppression of ferroelectricity. The paraelectric phase that results, found for intermediate temperatures and high magnetic fields, is characterized by a simple commensurate antiferomagnetic ordering.

We thank Peter Smeibidl, Sebastian Gerischer, Klaus Kiefer, and Michael Meissner from BENSC for their assistance during the experiment. We thank T. Kimura for sharing some of his unpublished results with us.
${ }^{1}$ N. Hill, J. Phys. Chem. B 104, 6694 (2000).

${ }^{2}$ T. Kimura, T. Goto, H. Shintani, K. Ishizaka, T. Arima, and Y. Tokura, Nature (London) 426, 55 (2003).

${ }^{3}$ T. Kimura, S. Ishihara, H. Shintani, T. Arima, K. T. Takahashi, K. Ishizaka, and Y. Tokura, Phys. Rev. B 68, 060403(R) (2003).

${ }^{4}$ J. Goodenough, Phys. Rev. 100, 564 (1955).

${ }^{5}$ T. Goto, T. Kimura, G. Lawes, A. P. Ramirez, and Y. Tokura, Phys. Rev. Lett. 92, 257201 (2004).

${ }^{6}$ T. Kimura, G. Lawes, T. Goto, Y. Tokura, and A. P. Ramirez, Phys. Rev. B 71, 224425 (2005).

${ }^{7}$ M. Kenzelmann, A. B. Harris, S. Jonas, C. Broholm, J. Schefer, S. B. Kim, C. L. Zhang, S.-W. Cheong, O. P. Vajk, and J. W. Lynn, Phys. Rev. Lett. 95, 087206 (2005).
${ }^{8}$ M. B. Walker, Phys. Rev. B 22, 1338 (1980).

${ }^{9}$ R. Kajimoto, H. Yoshizawa, H. Shintani, T. Kimura, and Y. Tokura, Phys. Rev. B 70, 012401 (2004).

${ }^{10}$ M. Mostovoy, Phys. Rev. Lett. 96, 067601 (2006).

${ }^{11}$ N. Aliouane, D. N. Argyriou, J. Strempfer, I. Zegkinoglou, S. Landsgesell, and M. v. Zimmermann, Phys. Rev. B 73, 020102(R) (2006).

${ }^{12}$ H. Katsura, A. V. Balatsky, and N. Nagaosa, cond-mat/0602547 Phys. Rev. Lett. (to be published).

${ }^{13}$ D. Senff, P. Link, K. Hradil, A. Hiess, L. Regnault, Y. Sidis, N. Aliouane, D. Argyriou, and M. Braden, cond-mat/0610620 (unpublished). 